Royal Brisbane and Women's Hospital, and ${ }^{4}$ Discipline of Molecular and Cellular pathology, The University of Queensland, Australia

Fine needle aspiration cytology is often the first or second line of investigation in the presence of enlarged thyroid gland or thyroid nodule, with a high sensitivity and specificity for neoplasms. Changes described as WHAFFT have been identified in subsequent histopathological examination. These range from nuclear pseudoinclusions mimicking papillary carcinoma, pseudoinvasion of thyroid capsule, infarction and vascular proliferation mimicking haemangiomas or papillary endothelial hyperplasia. Awareness of these changes may help to alleviate some of the stresses of diagnostic histopathological work. We present a recent case in which a similar change was identified in a 70 year old male with a $6 \mathrm{~mm}$ diameter nodule in the right lobe of thyroid. A fine needle aspiration cytology was reported as atypical follicular cells (Bethesda Category 3). Six weeks later, a right hemithyroidectomy was performed which showed a papillary microcarcinoma. A nodule of high vascularity and a needle tract were noted in the adjacent tissue. The differential diagnosis of such a nodule and ancillary tests aided in arriving at a final diagnosis is discussed.

\section{TRIPLICATION OF THE APPENDIX: A CASE REPORT}

Annie H. Jo, Brooke Beardsley

Canterbury Health Laboratories, Christchurch, New Zealand

Introduction: Duplication of the appendix has an incidence of $0.004 \%$. From 1892, cases have been reported in the literature, and have led to the Cave-Wallbridge classification system. Appendiceal triplications are rarer still, with only two cases of appendiceal triplication having been described in the literature. This is the first reported case of a single appendix with triplication of the lumen, each, at least focally, with its own separate muscular layer.

Case: A male aged 21 presented with abdominal pain. Examination revealed tenderness and guarding in the right iliac fossa. White count was normal, with CRP 34. Laparoscopic was converted to open appendicectomy as the inflamed appendix was adherent to terminal ileum and abdominal wall.

Pathology: Macroscopically, the base of the appendix had one lumen. This divided into a second adjacent lumen which split again resulting in three distinct lumens spanning a few transverse sections. The lumens then merged back into two, then into a single lumen at the tip.

Microscopically, three separate lumens were confirmed. Focally, each lumen had its own mucosa, submucosa, and muscularis propria with surrounding fibrofatty tissue. Acute appendicitis was also confirmed.

Discussion: These three cases of triplication could expand upon the Cave-Wallbridge classification for appendiceal duplication. The 1968 case describes a Type B triplication with three separate appendices arising from a single caecum. Our case and the 2006 case are variations of Type A. Our case has three lumens, focally with separate muscular layers, which extend partially through the length of the appendix. The other case had three lumens separated by submucosa within the same muscular layer, which extended fully through the length of the appendix. This case broadens the diversity of variations described in appendiceal triplications.

\section{BRAF (V600E) AND NRAS (Q61R/Q61L) MUTATION ANALYSIS USING IMMUNOHISTOCHEMISTRY: IMPLICATIONS FOR ROUTINE MUTATION TESTING OF MELANOMA SAMPLES}

Hojabr Kakavand $^{1,2}$, Trina Lum ${ }^{3}$, James S. Wilmott ${ }^{1,2}$, Elizabeth Smith $^{1,2}$, Sandra O'Toole ${ }^{1,3}$, Georgina V. Long ${ }^{1,2}$, Richard A. Scolyer ${ }^{1,2,3}$

${ }^{1}$ Sydney Medical School, the University of Sydney, Camperdown, ${ }^{2}$ Melanoma Institute Australia, North Sydney, and ${ }^{3}$ Tissue Pathology and Diagnostic Oncology, Royal Prince Alfred Hospital, Camperdown, NSW, Australia

Targeted therapies, such as BRAF and MEK inhibitors, have significantly improved the outcome of patients with metastatic melanoma. The appropriate administration of these therapies is dependent on the presence of mutations such as the BRAFV600E (prevalence 30-35\%), NRAS Q61R (10\%) and NRAS Q61L (5\%). There are various methods for mutation testing of samples each of which has varying sensitivities, costs and time allocations. The aim of this study was to assess the sensitivity and specificity of $B R A F$ and NRAS antibodies in a predefined cohort of patients with known mutation status. Three hundred and two metastatic melanoma samples with known mutation status using the Oncocarta panel (Sequenom) were included in this study. There were 92 patients with the BRAFV600E mutation, 28 with the BRAFV600K mutation, 50 with the NRASQ61R mutation, 22 with the NRASQ61L mutation, 20 with the NRASQ61K mutation and 90 that were wild type for both $B R A F$ and NRAS. Tissue microarrays were constructed and all samples were analysed using immunohistochemistry (IHC) for the BRAFV600E (VE1Spring Bioscience), NRASQ61R (SP174-Spring Bioscience) and NRASQ61L (26193-NewEast Biosciences) mutations. The staining was found to be homogeneous and cytoplasmic for all three antibodies. The sensitivity and specificity of each of the antibodies was analysed. The use of IHC is a fast, efficient and cost effective method to routinely screen patients for specific mutations. This will help facilitate the timely treatment of metastatic melanoma patients with targeted therapies.

\section{PRIMARY RETROPERITONEAL MUCINOUS CYSTADENOMA WITH BORDERLINE MALIGNANCY}

Laurne Kalinowski $^{1,3}$, Sally Byford ${ }^{2}$, Joanna Perry-Keene ${ }^{1}$ ${ }^{1}$ Department of Anatomical Pathology, ${ }^{2}$ Department of Obstetrics and Gynaecology, Nambour Base Hospital, Nambour, and ${ }^{3}$ Department of Pathology, University of Queensland, Brisbane, Qld, Australia

Primary retroperitoneal mucinous cystadenomas are rare with only seventeen cases previously reported in the literature with borderline features of malignancy. Primary retroperitoneal mucinous cystadenomas arise within the retroperitoneum without any connection to other organs and whilst predominantly occurring in women, cases are also described in men. We report a case in a 45-year-old woman who presented with an abdominal mass presumed ovarian in origin and benign on investigation. However, intra-operatively the mass was found to be retroperitoneal and histologically demonstrated borderline features. This case report includes a brief review of the literature examining primary retroperitoneal mucinous cystadenomas with borderline features and aims to highlight the 